\title{
Caracterización epidemiológica de hidatidosis humana y animal en la Región de Los Ríos, 1999-2009
}

\author{
Gerardo Acosta-Jamett, Reinaldo Vargas y Santiago Ernst
}

\section{Epidemiological description of human and animal hydatidosis in Los Ríos region, 1999- 2009}

Objective: To determine the prevalence of hydatidosis in humans and livestock in the Los Ríos region during the years 1999-2009. Methods: A retrospective survey of all discharge records of patients undergoing removal of cysts produced by Echinococcus granulosus during the period 1999-2009 in the Hospital Clinico Regional of Valdivia was conducted. With the information provided incidence rate, mortality rate and fatality rate were calculated. Additionally, with the aim of determining prevalence of hydatidosis in livestock (i.e. cattle, sheep and pigs) official records of slaughtered animals for the same period from the Ministry of Health of Los Ríos region were searched. Results: During the ten-year period 238 new human cases were recorded, the average incidence rate for the period was 6.1 per 100,000 inhabitants, the mortality rate was 0.1 per 100,000 inhabitants, and the fatality rate was $1.3 \%$. The most affected class group was the $15-64$ years old with a $70 \%$ of cases. The districts more affected were Valdivia and Río Bueno. Most cases (54\%) were from patients from rural areas, while the occupation more affected was housekeeping (29\%). The liver (56\%) and lungs (33\%) were the localization where cysts were most frequently found. On $55 \%$ of cases, patients did not show any complication; meanwhile, infected cyst (11\%) and unspecified complicated (8\%) were the more frequent complication. Overall, $89 \%$ of cases recovered totally after surgery. Finally, the average in patient time was 14.7 bed-days. Prevalence of hydatidosis in livestock was $23 \%$ in cattle, $23 \%$ in sheep and $12 \%$ in pigs. The highest prevalence was found in slaughter houses in the districts of Panguipulli and San José de la Mariquina. Discussion: Human and animal hydatidosis is highly prevalent in humans and livestock in Los Ríos region compared to national and international records. Further research is recommended to assess risk factors for human infection and improving record-keeping and trazability of slaughtered animals to implement control measures at the farm level to help to stop the life cycle of the parasite and thus reduce human infections.

Key words: Zoonosis, neglected diseases. Echinococcus granulosus, hydatidosis, cystic echinococcosis, hospital discharges.

Palabras clave: Zoonosis, enfermedades desatendidas, Echinococcus granulosus, equinococosis quística, hidatidosis, egresos hospitalarios.

\section{Introducción}

$\mathrm{H}$ idatidosis o equinococosis quística se denomina a la infección de animales herbívoros o del hombre con la forma larval o metacéstodo pertenecientes al género Echinococcus, mientras que equinococosis es la infección principalmente del perro con el parásito adulto ${ }^{1}$ y otros cánidos ${ }^{2}$. E. granulosus está ampliamente distribuido en el mundo y es endémico del sur de América del Sur, principalmente en Argentina, Chile, Perú y Uruguay ${ }^{1-3}$.

La estrecha convivencia entre el hombre y el perro, así como las prácticas deficientes de higiene personal y del entorno, unido al escaso conocimiento del hombre frente al riesgo al que se expone, debido a la falta de educación sanitaria asociada generalmente a bajos niveles culturales y/o económicos, generan las condiciones favorables para la transmisión de este parásito, existiendo una asociación inversamente proporcional entre el grado de educación y el riesgo de contagio ${ }^{1,4}$. El hombre participa activamente en la creación de condiciones ecológicas favorables para el desarrollo del ciclo biológico, mediante la alimentación de perros con vísceras crudas parasitadas, faenamiento clandestino de animales y abundancia excesiva de población canina. La transmisión del parásito al hombre, se produce por la ingestión inadvertida de huevos eliminados en las fecas de perros infectados, al acariciarlos, o por consumo de agua, verduras y hortalizas contaminadas ${ }^{3}$.

En el hombre, los quistes de E. granulosus pueden
Universidad Austral, Valdivia, Chile.

Facultad de Ciencias Veterinarias. Instituto de Medicina Preventiva Veterinaria (GAJ, RV) Universidad Iberoamericana de Ciencias y Tecnología. Facultad de Recursos Naturales y Ciencias Silvoagropecuarias (SE).

Financiamiento:

Conflictos de interés:

Recibido: 11 de marzo de 2015 Aceptado: 11 de junio de 2016

Correspondencia a:

Gerardo Acosta-Jamett gerardo.acosta@uach.cl 
demorar varios años en producir síntomas clínicos ${ }^{2}$. Muchos quistes son asintomáticos durante toda la vida del individuo infectado y constituyen, a veces, un hallazgo de autopsia, de una intervención quirúrgica o de radiografías realizadas por otras causas ${ }^{2}$. Moro y $\mathrm{Schant}^{3}$ mencionan que el rango de crecimiento de los quistes es variable, desde $1 \mathrm{a} 5 \mathrm{~cm}$ de diámetro por año e incluso, aunque la infección puede ser adquirida en la infancia, la mayoría de casos de hidatidosis en hígado y pulmón llegan a ser sintomáticos y diagnosticados recién en pacientes adultos $^{2}$. Se describe a la hidatidosis como una enfermedad de resolución quirúrgica ${ }^{5}$. La importancia médica y social de la hidatidosis radica en los daños que produce al enfermo y a la comunidad, ya que al tratarse de una afección crónica de larga evolución, disminuye la capacidad de trabajo de la persona afectada antes, durante y después del diagnóstico y tratamiento, más aún cuando éstos exigen una serie de exámenes clínicos y de laboratorio, la mayoría de los cuales son de elevado costo ${ }^{2,3}$. Esta enfermedad causa, además, importantes pérdidas económicas por las consecuentes ausencias en días laborales no trabajados y también en los costos productivos que provoca el decomiso de vísceras de ganado infectadas ${ }^{6,7}$.

De manera similar a lo que se reporta en otros países, $E$. granulosus genotipo G1 es la variante que principalmente se ha reportado en Chile ${ }^{8}$, cuyo hospedero definitivo es el perro y otros cánidos, posiblemente zorros ${ }^{9}$, mientras que el ganado doméstico actúa como hospedero intermediario $^{10,11}$. En el país, la notificación de hidatidosis durante los años 2000-2005 fue en promedio de 330 casos anuales, con una tasa de incidencia de 2,2 por 100 mil habs.; sin embargo, al comparar las cifras de notificación con las causales de egresos hospitalarios, se detectó que existe entre 66 y $75 \%$ de subnotificación, por lo que se estima que los casos nuevos fluctúan entre 970 y 1.320 casos anuales ${ }^{5}$. Por otro lado, Martínez ${ }^{12}$ reporta una incidencia de 1,9 casos por 100 mil habs. para el período 2001-2009 a nivel nacional.

La Región de Los Ríos es una región recientemente creada como tal en el año 2007. Cuenta con una población humana aproximada de 350.000 personas y sus principales actividades productivas están en la silvicultura, ganadería y turismo. Esta región tiene índices de pobreza superiores a la media nacional y cuenta con población viviendo en zonas rurales cercana a $30 \%{ }^{13}$, lo cual podría incidir en alta incidencia de hidatidosis en personas.

Aunque los estudios de Cortés y Valle y Martínez $^{12}$ analizan las estadísticas globales de la Región de Los Ríos, el presente trabajo tiene por objetivo aportar información sobre la morbilidad, mortalidad y letalidad por hidatidosis humana en esta región durante los años 1999-2009 y adicionalmente, determinar la prevalencia de hidatidosis en bovinos, ovinos y porcinos, beneficiados en mataderos de esta región, durante el mismo período.

\section{Material y Métodos}

\section{Hidatidosis humana}

Con la finalidad de determinar la incidencia de hidatidosis en humanos, se realizó una revisión de las fichas clínicas de los pacientes operados por hidatidosis registrados en el Servicio de Orientación Médico Estadístico (S.O.M.E.) del Hospital Clínico Regional de Valdivia, durante el período 1999-2009. Los datos utilizados en este trabajo corresponden a las estadísticas de la Provincia de Valdivia (Región de Los Lagos), actual Región de los Ríos, constituida en octubre del año 2007. Con la información registrada se cuantificó la morbilidad, mortalidad y letalidad por hidatidosis de la siguiente forma:

A) Tasa de incidencia: Número de casos nuevos de hidatidosis confirmados quirúrgicamente durante el período de un año, dividido por el total de la población expuesta a riesgo (según Censo 2002) ${ }^{13}$, expresado como el número de casos anuales por 100 mil habs.

B) Tasa de mortalidad: Número de defunciones ocurridas en los casos nuevos de hidatidosis confirmados quirúrgicamente durante el período de tiempo de un año, dividido por el total de la población expuesta a riesgo, expresado como el total de defunciones anuales por cien mil habitantes.

C) Tasa de letalidad: Número de defunciones ocurridas en los casos nuevos de hidatidosis confirmados quirúrgicamente durante el período de tiempo de un año, dividido por el total de casos nuevos registrados durante ese mismo período, expresado como el número de defunciones anuales por cada cien casos de hidatidosis.

Además, la información extraída de las fichas clínicas fue clasificada según sexo, edad, ocupación, comuna, lugar de habitación (urbano o rural), localización del quiste, condición del quiste al momento de ser operado y condición al egreso y período de hospitalización.

Los pacientes mejorados del todo son aquellos que egresaron del hospital sin el o los quistes diagnosticados. Los pacientes recuperados parcialmente engloban casos que después de haber sido diagnosticados y operados de aquel(los) quiste(s) que indujera(n) la sintomatología, aún persiste en ellos algún(os) quiste(s) que no comprometen mayormente su estado de salud y que obedecen en su mayoría a casos de siembra hidatídica. Los casos no recuperados son aquellos en que después del acto quirúrgico permanece en ellos algún quiste que está comprometiendo el estado de salud del paciente y que por diversos motivos no pudo ser extraído. En algunos casos se requerirá de una nueva intervención quirúrgica o un tratamiento medicamentoso prolongado. Los casos fallecidos corresponden a aquellos que murieron durante el período de hospitalización. 
Los resultados anteriores se dividieron por 11 años, obteniéndose tasas promedios anuales para el período en estudio.

\section{Hidatidosis en animales}

La determinación de prevalencia de hidatidosis animal en mataderos de la actual Región de Los Ríos correspondiente al período de años 1999-2009 se realizó a través del estudio de los datos referentes a la frecuencia de presentación de quistes en vísceras decomisadas, obtenidos de los informes mensuales y anuales de causales de decomiso en mataderos, disponibles en la Secretaría Regional Ministerial (SEREMI) de Salud de la Región de Los Ríos (Figura 1). A partir de los datos obtenidos se confeccionaron tablas de frecuencia según especie faenada.

\section{Análisis de datos}

Toda la información fue trasladada a planillas del programa Microsoft Excel 2007 para su análisis estadístico descriptivo. Se utilizó prueba de $\chi^{2}$ en programa estadístico R 2.13.1 para determinar diferencias entre proporciones $^{14}$.

\section{Resultados}

\section{Hidatidosis en humanos}

Durante los años 1999-2009, se operaron 284 casos de hidatidosis, de los cuales 238 correspondieron a casos nuevos y 46 a re-hospitalizaciones. La incidencia para el período fluctuó entre 3,6 y 8,4 por 100 mil habitantes, mientras la incidencia promedio para el período fue de 6,1 casos por 100 mil habs. Además, durante este período se produjeron tres defunciones, determinando una tasa de mortalidad promedio de 0,1 por 100 mil habs. $y$ una tasa de letalidad de 1,3\% (Tabla 1). A pesar de observarse una variación en las tasas de incidencias anuales se observó una tendencia a su disminución en el período en estudio (Figura 2).

En la Figura 3 se puede apreciar que 125 casos, equivalentes al $53 \%$ del total de los casos nuevos fueron hombres y 113 mujeres (47\%). Sin embargo, esta diferencia no fue significativa $\left(\chi^{2}=0,47 ; \mathrm{df}=1 ; \mathrm{p}=0,49\right)$. La tasa promedio anual en el período de estudio para el sexo masculino fue de 6,4 por 100 mil habs., mientras que para el sexo femenino fue de 5,8 por 100 mil habs., no existiendo diferencias entre sexos. El grupo de edad más afectado fue el comprendido entre los 15 y 64 años, encontrándose diferencias significativas entre éste y los otros grupos etários $\left(\chi^{2}=6,93 ; \mathrm{df}=2 ; \mathrm{p}=0,03\right)$ (Figura 3 ).

De los 238 casos nuevos, se observó que la ocupación más frecuentemente afectada fue dueña de casa (29\%), seguido de los estudiantes (16\%) y trabajador del sector agropecuario (14\%). Estas tres ocupaciones sumaron un total de 154 casos nuevos representando $65 \%$ del total de casos (Figura 4). En la Figura 5 se observa que la mayoría de los pacientes operados prevenían de las comunas de Valdivia con 54 casos (23\%), Panguipulli con 37 casos (16\%) y Lanco con 27 casos (11\%). Las tasas promedios

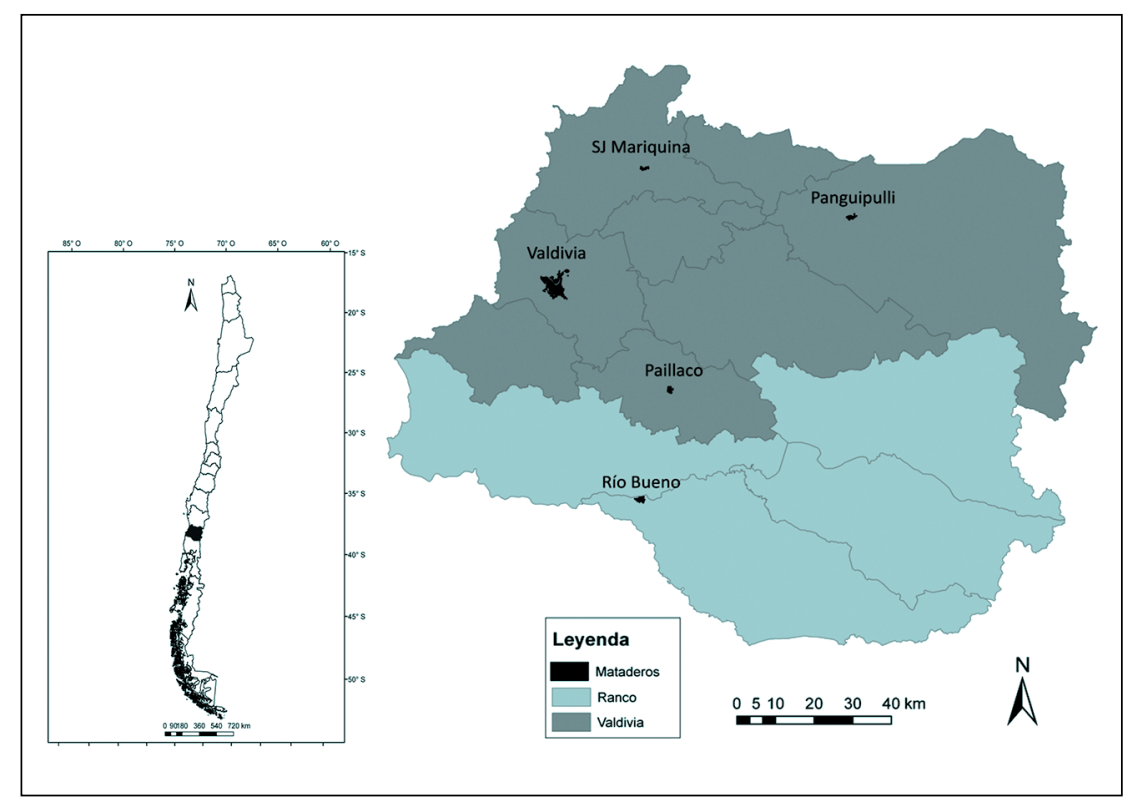

Figura 1. Mapa de Chile destacando la Región de Los Ríos (a la izquierda). A la derecha se observa las dos provincias de la Región en diferente color y las comunas son delimitadas en gris. Los mataderos donde se obtuvo información del decomiso de los animales se indican en negro.

Tabla 1. Descripción del número de casos, número de fallecidos y tasa de mortalidad (por 100.000 habs.) y de letalidad (\%) por hidatidosis humana según registros de egresos hospitalarios del Hospital Regional de Valdivia en la Región de Los Ríos en el período 1999-2009

\begin{tabular}{|ccccc}
\hline Años & n casos & $\begin{array}{c}\text { n de } \\
\text { fallecidos }\end{array}$ & $\begin{array}{c}\text { Tasa de } \\
\text { mortalidad }\end{array}$ & $\begin{array}{c}\text { Tasa de } \\
\text { letalidad }\end{array}$ \\
\hline 1999 & 27 & 1 & 0,3 & 3,7 \\
\hline 2000 & 19 & 0 & 0,0 & 0,0 \\
\hline 2001 & 28 & 1 & 0,3 & 3,6 \\
\hline 2002 & 30 & 0 & 0,0 & 0,0 \\
\hline 2003 & 23 & 0 & 0,0 & 0,0 \\
\hline 2004 & 18 & 0 & 0,0 & 0,0 \\
\hline 2005 & 20 & 0 & 0,0 & 0,0 \\
\hline 2006 & 18 & 0 & 0,0 & 0,0 \\
\hline 2007 & 18 & 0 & 0,0 & 0,0 \\
\hline 2008 & 24 & 1 & 0,3 & 4,2 \\
\hline 2009 & 13 & 0 & 0,0 & 0,0 \\
\hline Total & 238 & 3 & 0,1 & 1,3 \\
\hline
\end{tabular}



dencia anual de hidatidosis humana según egresos hospitalarios en el período comprendido entre 19992009 en la Región de Los Ríos.
Figura 2. Inci-
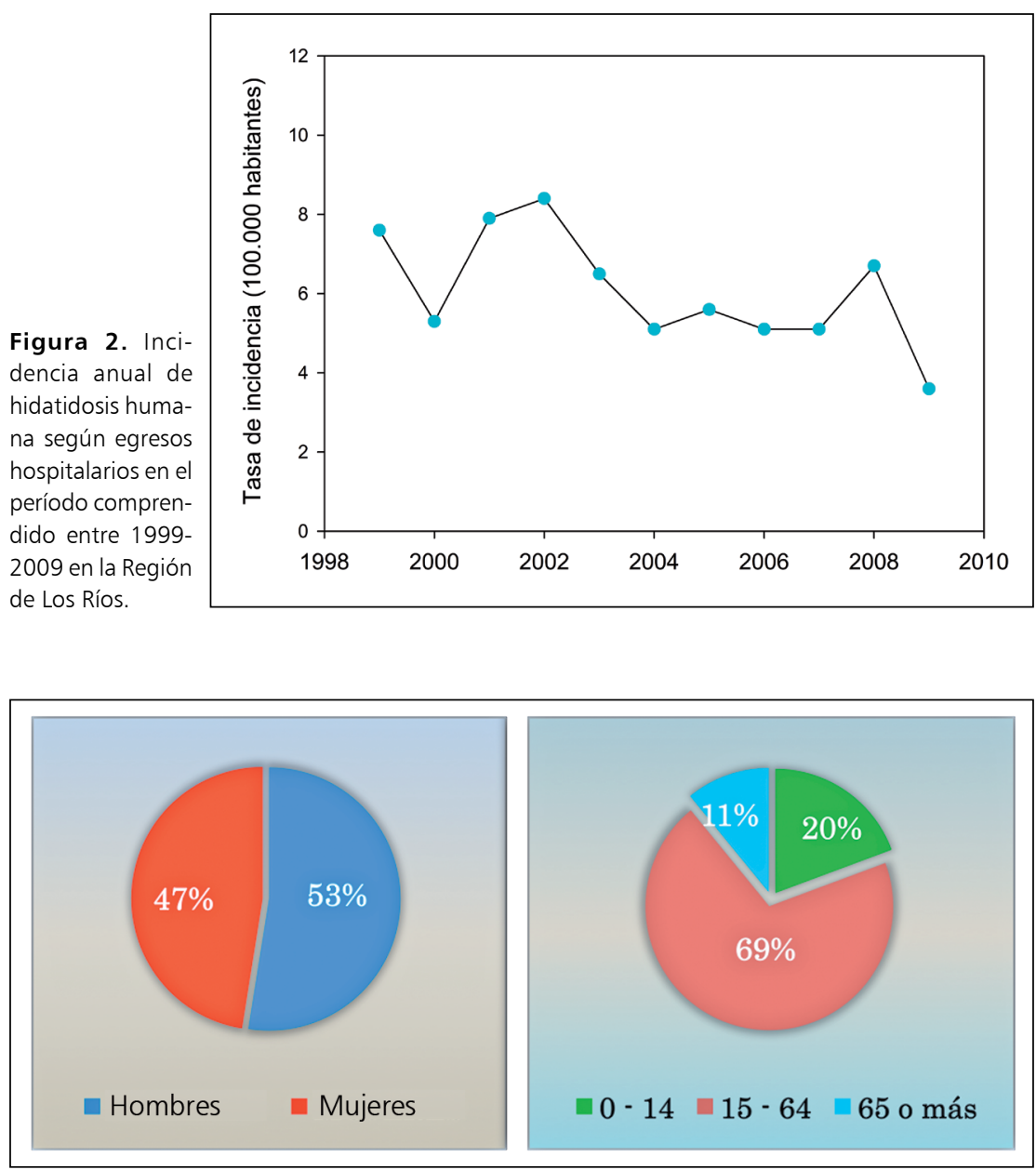

Figura 3. Distribución porcentual de casos de hidatidosis en humanos según sexo (izquierda) y edad (derecha) de los afectados en el período 1999-2009 en la Región de Los Ríos. anuales más altas por comuna se registraron en Lanco con 16, Panguipulli con 10 y Los Lagos con 9 casos por 100.000 habs., respectivamente.

Al analizar si existía una diferencia entre el origen urbano o rural de los pacientes operados por primera vez, se detectó que 129 casos (54\%) tenían procedencia rural, mientras la condición urbana se presentó en 107 casos $(45 \%)$, siendo estas diferencias significativas $\left(\chi^{2}=56,3\right.$; $\mathrm{df}=1 ; \mathrm{p}<0,0001)$. Los dos casos restantes $(0,8 \%)$ corresponden a aquellos que no especifican la condición urbano-rural de su procedencia. A nivel comunal, sólo Valdivia, La Unión y Máfil presentaron un predominio urbano, donde las tasas más altas fueron Lanco y Lago Ranco, mientras que las tasas rurales más altas se encontraron en Lanco, nuevamente y Corral. La tasa de 10,4 por 100 mil habs. para el área rural de la Región de Los Ríos es considerablemente mayor a la tasa para la condición urbana que fue de 4,0 por 100 mil habs. Se precisa que estas tasas corresponden a valores promedios anuales del período para la distribución urbano-rural de cada comuna (Figura 5).

Según la localización del quiste al momento de ser operado, $56 \%$ presentó sólo quistes de ubicación hepática y $33 \%$ sólo pulmonar, mientras la presentación de quistes en ambos órganos fue de 6\%. El resto de los quistes tuvieron distintas localizaciones (Figura 6). De los casos nuevos, 130 pacientes (55\%) estaban afectados por quistes íntegros, no complicados, potencialmente patógenos. Los quistes clasificados como inocuos (muerto, calcificado o vomicado) se presentaron en $9 \%$ con un total de 21 casos; el $37 \%$ restante corresponde a 87 casos, que tuvieron diversas complicaciones, de las cuales las más frecuentes fueron infectado (11\%) y complicado sin

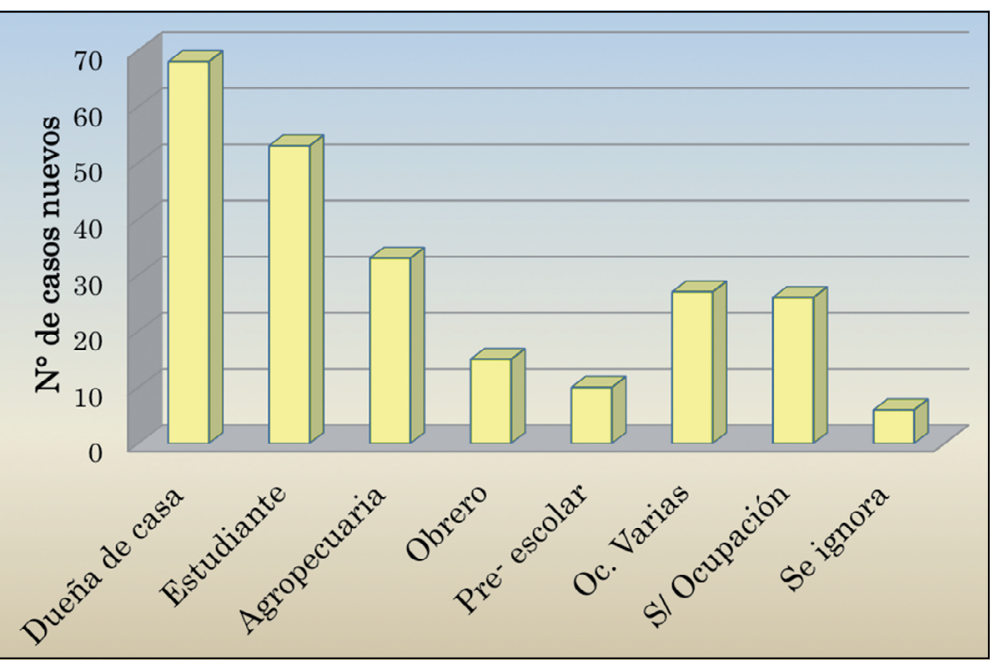

Figura 4. Frecuencia de los casos de hidatidosis humana según ocupación operados en el período comprendido entre 1999 al 2009 en la Región de Los Ríos.

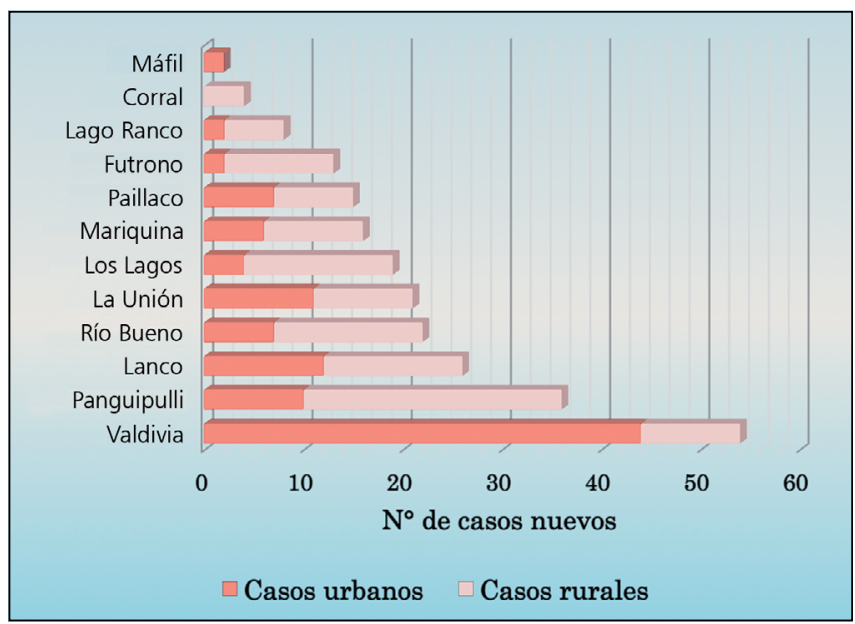

Figura 5. Frecuencia de casos de hidatidosis reportada por egresos hospitalarios según comuna y lugar (urbano o rural) de residencia del paciente durante el período 1999 al 2009 en la Región de Los Ríos. 
especificar ( $8 \%$ ). Con respecto a la condición al egreso del hospital de los casos nuevos, se determinó que 212 $(89 \%)$ egresaron recuperados totalmente, 17 (7\%) lo hicieron parcialmente, $6(3 \%)$ no se recuperaron y 3 $(1,3 \%)$ fallecieron. Durante los 11 años que comprende el estudio, los casos nuevos significaron en conjunto 3.495 días de hospitalización, con un promedio para el período de 14,7 días-cama de hospitalización por paciente (rango: 2-75 días) (Tabla 2).

\section{Hidatidosis en animales}

Durante el período en estudio se determinó que $23 \%$ de los bovinos, $23 \%$ de los ovinos y $12 \%$ de los porcinos faenados en todos los mataderos de la Región de Los

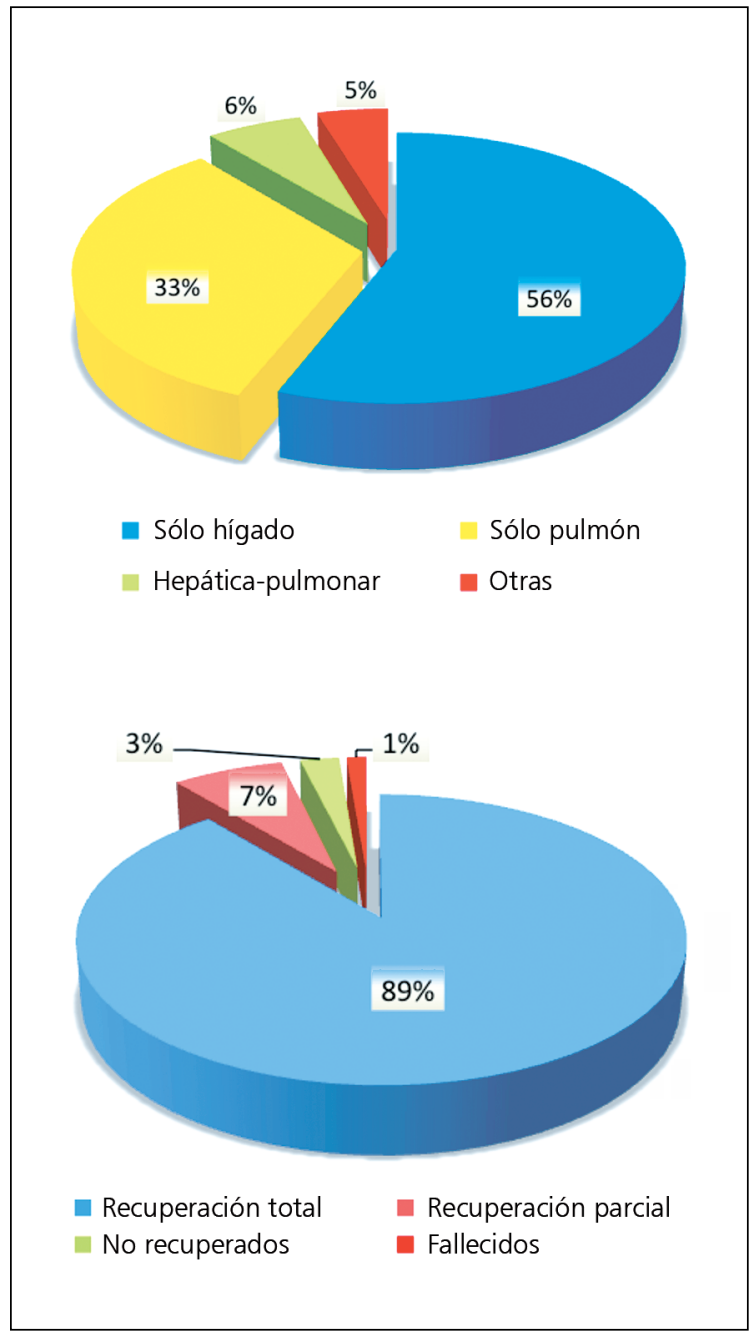

Figura 6. Distribución porcentual de la ubicación de los quistes operados (arriba) y condición al egreso (abajo) de los pacientes operados por hidatidosis durante el período 1999 al 2009 en la Región de Los Ríos.
Ríos, se encontraban afectados por hidatidosis, siendo estas diferencias significativas y dadas principalmente por la menor prevalencia de hidatidosis en cerdos que en las otras dos especies $\left(\chi^{2}=4.182 ; \mathrm{df}=2 ; \mathrm{p}<0,0001\right)$. En la Tabla 3 se puede apreciar que Panguipulli registró la prevalencia más alta en bovinos $(90,3 \%)$, así como en porcinos $(81 \%)$, mientras San José de la Mariquina presentó la mayor prevalencia en ovinos $(85 \%)$. La prevalencia animal en la Región de Los Ríos para todas las especies beneficiadas durante el período en estudio fue de $22 \%$.

Tabla 2. Condición al egreso y días de hospitalización de los casos de hidatidosis operados durante el período 1999 al 2009, en la Región de Los Ríos

\begin{tabular}{|ccccc}
\hline Años & n casos & $\begin{array}{c}\mathbf{n} \text { casos recuperados } \\
\text { totalmente }\end{array}$ & $\begin{array}{c}\text { n recuperados } \\
\text { parcialmente }\end{array}$ & $\begin{array}{c}\text { Días de hospitalización } \\
\text { promedio }\end{array}$ \\
\hline 1999 & 27 & 23 & 2 & 10,5 \\
\hline 2000 & 19 & 18 & 1 & 14,7 \\
\hline 2001 & 28 & 25 & 2 & 13,6 \\
\hline 2002 & 30 & 21 & 5 & 16,0 \\
\hline 2003 & 23 & 18 & 2 & 13,8 \\
\hline 2004 & 18 & 18 & 0 & 17,9 \\
\hline 2005 & 20 & 15 & 2 & 16,7 \\
\hline 2006 & 18 & 17 & 2 & 12,0 \\
\hline 2007 & 18 & 21 & 1 & 12,6 \\
\hline 2008 & 24 & 13 & 0 & 15,8 \\
\hline 2009 & 13 & 212 & 0 & 21,3 \\
\hline Total & 238 & & 17 & 14,7 \\
\hline
\end{tabular}

Tabla 3. Registro de ganado cuyas vísceras fueron decomisadas por hidatidosis en mataderos de la Región de Los Ríos, para el período 1999-2009

\begin{tabular}{|c|c|c|c|c|c|c|}
\hline \multirow[t]{2}{*}{ Mataderos } & \multicolumn{2}{|c|}{ Bovinos } & \multicolumn{2}{|c|}{ Ovinos } & \multicolumn{2}{|c|}{ Porcinos } \\
\hline & $\begin{array}{l}\text { Examina- } \\
\text { dos }\end{array}$ & $\begin{array}{c}\text { Infectados } \\
(\%)\end{array}$ & $\begin{array}{l}\text { Examina- } \\
\text { dos }\end{array}$ & $\begin{array}{c}\text { Infectados } \\
\text { (\%) }\end{array}$ & $\begin{array}{l}\text { Examina- } \\
\text { dos }\end{array}$ & $\begin{array}{c}\text { Infectados } \\
(\%)\end{array}$ \\
\hline Valdivia & 535.916 & 21,1 & 26.810 & 25,6 & 33.586 & 12,5 \\
\hline Río Bueno & 63.724 & 34,0 & 8.001 & 14,3 & 30.096 & 9,8 \\
\hline S. J. Mariquina & 7.356 & 69,8 & 127 & 85,0 & 637 & 60,3 \\
\hline Panguipulli' & 3.014 & 90,3 & 65 & 73,8 & 880 & 80,5 \\
\hline Paillaco $^{2}$ & 6.602 & 23,2 & 1.311 & 3,4 & 1.522 & 3,7 \\
\hline Total & 616.612 & 23,4 & 36.314 & 22,6 & 66.721 & 12,4 \\
\hline
\end{tabular}




\section{Discusión}

La tasa de incidencia promedio anual para el período estudiado de 6,1 por 100 mil habs. es superior a la tasa de 2,2 por 100 mil habs. reportada para los años 2000-2005 a nivel nacional, lo cual coincide con que la Región de Los Ríos sería una de las más afectadas por esta enfermedad en el país ${ }^{5,12}$. La tendencia a la disminución en la incidencia es similar a la determinada según egresos hospitalarios, para los años 2000-2005 5

\section{Distribución por grupo etario}

En Chile, Cortés y Valle ${ }^{5}$ determinaron al rango etario de 30 a 59 años, como el más afectado, con un promedio de edad de los casos notificados de 38 años. Lo anterior coincide con lo reportado en nuestro estudio y otros estudios a nivel mundial ${ }^{15-21}$, donde se observó una gran cantidad de casos en el grupo de adultos. En este sentido, Schenone y cols ${ }^{22}$, señalan que en esta enfermedad, como también sucede con otras infecciones parasitarias crónicas, las tasas de positividad aumentan paralelamente con la edad de los individuos infectados. No obstante lo anterior, es importante destacar el gran porcentaje de niños y adolescentes ( $<14$ años de edad) con hidatidosis (11\%), lo cual es levemente superior a lo reportado a nivel nacional ${ }^{5}$ y muy superior a lo que se reporta en otras partes del mundo ${ }^{15-21}$, lo cual indica una alta tasa de infección en años recientes.

\section{Distribución por sexo}

Aunque en este estudio se observó una tendencia a una mayor prevalencia de hidatidosis en hombres que en mujeres, similar a lo reportado por Cortes y Valle ${ }^{5}$, esta diferencia no fue significativa. Dispares resultados se han observado en la literatura médica al comparar la prevalencia entre sexos, lo cual puede deberse a diversos factores de riesgo asociados a un mayor contacto con los perros en un sexo que el otro, lo cual puede variar entre países y/o regiones. Por ejemplo, Zhang y col..$^{15}$, reportaron una mayor prevalencia de hidatidosis en mujeres $(60 \%)$ que en hombres (40\%) en dos hospitales en China, operadas entre 2004 y 2013, similar a lo reportado por Ernest y cols. ${ }^{21}$ en Tanzania. Por el contrario, López-Bernus y cols. ${ }^{16}$, reportaron una mayor prevalencia en hombres (57\%) en el área de Castilla-León en España en el período 2000-2012.

\section{Actividad laboral e hidatidosis}

La distribución por ocupación revela que las dueñas de casa presentaron la mayor frecuencia, seguidas por el grupo de los estudiantes y ocupación agropecuaria (Figura 4). Cabe señalar que la clasificación "agropecuaria", incluye diferentes labores vinculadas a esta categoría, como lo son trabajador agrícola, obrero de lechería y agricultor. Otros estudios también han reportado a las dueñas de casa como las más afectadas por esta enfer$\operatorname{medad}^{23}$. La mayor cantidad de casos registrados en las ocupaciones anteriormente mencionadas, tiene su posible explicación en la estrecha relación que existe entre el perro y su propietario, ya que en esta zona el perro no es utilizado preferentemente en labores relacionadas con el pastoreo, sino más bien es utilizado casi exclusivamente en labores de vigilancia de los hogares (observación personal). En este sentido, la dueña de casa, encargada de su alimentación y cuidado, y los estudiantes, que consideran al perro como mascota, son quienes tienen una relación más estrecha con el animal y presentan el mayor riesgo de contraer el parásito. Sin embargo, se debe considerar que siendo la hidatidosis una enfermedad crónica, las estadísticas sobre la ocupación actual del paciente no siempre son relevantes, debido a que estos pudieron haber ejercido actividades laborales que significaron un mayor riesgo de exposición en el pasado.

\section{Distribución geográfica}

Las comunas de Valdivia, Panguipulli y Lanco presentaron el mayor número de casos nuevos de hidatidosis operada (Figura 5). Esto podría ser explicado porque estas comunas presentan individualmente una de las mayores poblaciones dentro de la región y proporcionalmente corresponderían al mayor número de casos. Además, se debe considerar que si bien Valdivia aparece con la mayor frecuencia comunal, esto podría deberse al hecho de tener el centro hospitalario de mayor atracción de la zona, lo que podría responder a que pacientes atendidos en dicho centro hospitalario durante el tiempo de control médico, diagnóstico de la enfermedad y días de tratamiento, entregan como residencia temporal esta comuna, la que no necesariamente corresponde a su domicilio permanente.

De manera similar a lo reportado en este estudio, esta enfermedad se ha descrito como una afección que ocurre en condiciones de ruralidad ${ }^{1}$. Por ejemplo, en un estudio realizado en Bangladesh analizando registros de egresos hospitalarios, se encontró que sobre $70 \%$ de los pacientes habitaban en áreas rurales ${ }^{23}$. Del mismo modo, similares resultados se reportaron en un estudio realizado en España ${ }^{16}$. En Chile, Acosta-Jamett y col ${ }^{11}$, analizaron la prevalencia de hidatidosis humana y animal en la Región de Coquimbo; ellos señalan que las condiciones de vida en ambientes rurales están asociadas con un mayor riesgo de infección por E. granulosus, donde el alto contacto con perros, la recolección de fecas y la contaminación de huertas con fecas de perros, su escasa desparasitación y el faenamiento de ganado para auto-consumo en los hogares, son factores de riesgo para el contagio de los seres humanos con el parásito ${ }^{4}$. No obstante, en ciertos casos como lo detectado por Acosta-Jamett y cols. ${ }^{10}$, en algunas ciudades se dan también las condiciones que favorecen el 
contagio desde perros a personas, como faenamiento de ganado, lo cual podría estar ocurriendo en las comunas de Valdivia, La Unión y Máfil, donde se reporta una gran cantidad de casos de hidatidosis de personas originarias de zonas urbanas. Los contagios urbanos podrían deberse a la constante y mantenida migración desde sectores rurales a urbanos, sumado a la mantención de costumbres rurales como el faenamiento de animales en los hogares y el alimentar perros con vísceras contaminadas ${ }^{10,22}$. No obstante lo anterior, no se debe descartar que el contagio no haya ocurrido en las zonas urbanas, sino más bien en zonas rurales y esta descripción urbana se deba a la dirección que entregan los pacientes de zonas rurales mientras están en tratamiento (observación personal).

\section{Localizaciones anatómicas}

Al clasificar los casos de hidatidosis en humanos por localización del quiste, el mayor porcentaje registró una ubicación hepática exclusiva $(56 \%)$, seguido por la pulmonar exclusiva y pulmonar/hepática. Similares resultados se han reportado en estudios realizados en China ${ }^{15}$, Italia $^{18,19}$, Etiopia $^{17}$, Rumania ${ }^{20}$, Tanzania ${ }^{21}$ y Bangladesh ${ }^{23}$; no obstante, en estos estudios, a diferencia de lo encontrado en la Región de Los Ríos, se reportó que sobre 70\% de los quistes presentaban ubicación hepática. Cuál podría ser la explicación para la más baja presentación hepática en esta región de Chile se desconoce.

\section{Estado del quiste}

En el presente estudio, cerca de $9 \%$ de los casos presentaron quistes inocuos (muertos, calcificados o vomicados), que incluso podrían no haber sido intervenidos o correspondieron a un hallazgo de otra patología. El mayor número de pacientes estaba afectado por quistes íntegros no complicados (55\%), mientras que el $37 \%$ restante presentó diversas complicaciones, siendo las más frecuentes los quistes infectados (11\%) y complicados sin especificar (8\%). Complicaciones se han reportado también en la literatura médica, donde por ejemplo se reportó hasta $20 \%$ de complicaciones en pacientes operados por hidatidosis en Rumania ${ }^{20}$.

\section{Condición al egreso}

De los 238 casos nuevos de hidatidosis atendidos durante 1999-2009, 89\% del total de casos, se recuperó totalmente.

\section{Período de hospitalización}

El promedio de 14,7 días-cama por paciente obtenido en el presente trabajo es similar a lo obtenido en otros estudios $^{24}$. El desarrollo de nuevas técnicas quirúrgicas y el diagnóstico precoz, posibilitan una intervención quirúrgica temprana y con menores complicaciones; además de la complementación del tratamiento quirúr- gico con medicamentos, antes, durante y después de la intervención.

Si tomamos en cuenta las disminuciones de las tasas de mortalidad y letalidad en el período, esto podría deberse al incremento de la red de asistencia rural y el desarrollo de técnicas de diagnóstico precoz, lo cual permite una intervención quirúrgica temprana con menor riesgo de muerte. Lo anterior se confirma por el hecho de que un $55 \%$ de los pacientes presentaron quistes inocuos y no complicados, lo cual al momento de la intervención reduce significativamente los riesgos de muerte.

\section{Equinococosis quística en animales}

Los resultados de hidatidosis en animales son similares a los registrados por Acosta-Jamett y cols. ${ }^{11}$, en el norte de Chile, aunque según estos mismos autores, indican que estos estudios podrían introducir sesgos a su estimación, debido a la selección no aleatoria de los animales que se envían a los mataderos, sumado a la incapacidad de identificar quistes pequeños durante la inspección de las carcasas y la variabilidad en la detección entre los inspectores oficiales de los diferentes mataderos. Además, en este estudio no se incluye información respecto de la matanza domiciliaria, lo que claramente podría dar una estimación más confiable sobre la prevalencia de hidatidosis en la región. Las prevalencias reportadas en ganado en este estudio, por un lado son mayores a las reportadas en bovinos y ovinos que las reportadas en estudios realizados en Irán ${ }^{25}$, Sudán $^{26}, \mathrm{Kenia}^{27}$, Jordania ${ }^{28}$ y en Grecia ${ }^{29}$, y menores a lo reportado en Tanzania ${ }^{30}$. Aunque no se reportan en la literatura médica muchos estudios sobre decomisos en porcinos, lo encontrado en este estudio supera a lo reportado internacionalmente ${ }^{29}$.

La determinación de prevalencia de hidatidosis en animales, a través del estudio de decomisos en mataderos, junto al análisis de la incidencia de esta enfermedad en humanos, pueden ser utilizados como un punto de partida para futuros estudios epidemiológicos en la Región de Los Ríos. Adicionalmente, se podrían incluir estudios seroepidemiológicos y/o ultrasonográficos en humanos, así como la trazabilidad de los animales faenados y técnicas coproparasitarias en perros para obtener resultados más exactos sobre la prevalencia de esta enfermedad en esta región. Los resultados obtenidos indican que la hidatidosis es aún un problema importante de salud pública en la Región de Los Ríos, lo que debiera estimular el desarrollo de actividades de intervención para controlar esta enfermedad desatendida.

Agradecimientos. Agradecemos especialmente al personal del Servicio de Orientación Médico Estadístico (S.O.M.E.) del Hospital Clínico Regional de Valdivia, por su buena voluntad y servicio, especialmente a la Srta. Pamela Montiel V. 


\section{Resumen}

Objetivos y Métodos: Para determinar la prevalencia de hidatidosis humana y animal en la Región de Los Ríos durante los años 1999-2009, se realizó un análisis retrospectivo de las fichas clínicas de operados por esta enfermedad en el Hospital Clínico Regional de Valdivia. Se cuantificó morbilidad, mortalidad y letalidad y se determinaron algunas características epidemiológicas de la enfermedad. Adicionalmente, para determinar la prevalencia de equinococosis quística en el ganado, se analizaron los registros oficiales sobre la frecuencia de presentación de quistes en vísceras decomisadas en bovinos, ovinos y porcinos faenados en mataderos de la región durante el mismo período, obtenidos de la SEREMI de Salud. Resultados: Se registraron 238 casos nuevos en humanos con una tasa de incidencia promedio de 6,1 por 100 mil habs., una tasa de mortalidad de 0,1 por 100 mil habs. y una tasa de letalidad de 1,3\%. En total, 53\% de los casos fueron hombres y $47 \%$ mujeres. El grupo de edad entre 15 y 64 años fue el más afectado (70\% del total). Las comunas con mayor número de casos fueron Valdivia y Río Bueno. Se detectaron más casos (54\%) en zonas rurales que urbanas (46\%). Por otro lado, la ocupación más afectada correspondió a dueñas de casa. La localización más frecuente fue hepática exclusiva (56\%). Un 55\% de los pacientes no mostró complicación de los quistes tratados. Después de la operación, $89 \%$ de los casos se recuperó totalmente, mientras que el período de hospitalización promedio fue de 14,7 días-cama por paciente. La prevalencia de equinococosis en ganado fue de $23 \%$ en bovinos, $23 \%$ en ovinos y $12 \%$ en porcinos. Las mayores prevalencias se reportaron en mataderos de Panguipulli y San José de la Mariquina. Discusión: La hidatidosis humana y animal son altamente prevalentes en la Región de Los Ríos comparadas con el resto del país y otras naciones. Se recomienda la realización de estudios avanzados para determinar factores de riesgo para la infección en humanos y realizar mejoras en la trazabilidad de los animales sacrificados para implementar medidas de control a nivel del predio y de esa forma cortar el ciclo del parásito y ayudar a reducir la infección en humanos.

\section{Referencias bibliográficas}

1.- Otero-Abad B, Torgerson P R. A systematic review of the epidemiology of echinococcosis in domestic and wild animals. PLoS Negl Trop Dis 2013; 7 (6): e2249.

2.- Eckert J, Deplazes P. Biological, epidemiological, and clinical aspects of echinococcosis, a zoonosis of increasing concern. Clin Microbiol Rev 2004; 17 (1): 107-35.

3.- Moro P, Schantz P M. Echinococcosis: a review. Int J Infect Dis 2009; 13 (2): 125-33.

4.- Acosta-Jamett G, Weitzel T, Boufana B, Adones C, Bahamonde A, Abarca K, et al. Prevalence and risk factors for echinococcal infection in a rural area of northern Chile: a household-based cross-sectional study. Plos Neglect Trop Dis 2014; 8 (8): e3090.

5.- Cortés S, Valle C. Hidatidosis humana: generalidades y situación epidemiológica en Chile según egresos hospitalarios y notificación obligatoria entre los años 2001 y 2005 . Rev Chilena Infectol 2010; 27 (4): 329-35.

6.- Budke C M, Deplazes P, Torgerson P R. Global socioeconomic impact of cystic echinococcosis. Emerg Infect Dis 2006; 12 (2): 296-303.

7.- Moro P L, Budke C M, Schantz P M, Vásquez J, Santivañez S J, Villavicencio J. Economic impact of cystic echinococcosis in Perú. Plos Neglect Trop D 2011; 5 (5): e1179. doi:10.1371/journal.pntd.0001179.
8.- Manterola C, Benavente F, Melo A, Vial M, Roa J C. Description of Echinococcus granulosus genotypes in human hydatidosis in a region of southern Chile. Parasitol Int 2008; 57: 342-6.

9.- Acosta-Jamett G, Cleaveland S, Bronsvoort B M D, Cunningham A A, Bradshaw H, Craig P S. Echinococcus granulosus infection in foxes in Coquimbo distric, Chile. Arch Med Vet 2015; 47 (4): 409-13.

10.- Acosta-Jamett G, Cleaveland S, Bronsvoort B M D, Cunningham A A, Bradshaw $\mathrm{H}$, Craig P S. Echinococcus granulosus infection in domestic dogs in urban and rural areas of the Coquimbo region, north-central Chile. Vet Parasitol 2010; 169 (1-2): 117-22.

11.- Acosta-Jamett G, Cleaveland S, Bronsvoort B M D, Cunningham A A, Bradshaw H, Craig P S. Echinococcus granulosus infection in human and livestock in the Coquimbo region, north-central Chile. Vet Parasitol 2010; 169 (1-2): 102-10.

12.- Martínez P. Hidatidosis humana: antecedentes generales y situación epidemiológica en Chile, 2001-2009. Rev Chilena Infectol 2011; 28 (6): 585-91.

13.- INE. Instituto Nacional de Estadísticas. Censo 2002. Resultados población y vivienda. Santiago; 2005.

14.- R: A Language and Environment for Statistical Computing. Vienna, Austria: the R Foundation for Statistical Computing. Available online at http://www.R-project.org/ (2015).

15.- Zhang T, Zhao W, Yang D, Piao D, Huang S,
Mi Y, et al. Human cystic echinococcosis in Heilongjiang Province, China: a retrospective study. BMC Gastroenterol 2015; 15: 29.

16.- López-Bernus A, Belhassen-García M, Alonso-Sardon M, Carpio-Pérez A, Velasco-Tirado V, Romero-Alegria A, et al. Surveillance of human echinococcosis in Castilla-Leon (Spain) between 2000-2012. PLoS Negl Trop Dis 2015; 9 (10): e0004154.

17.- Assefa H, Mulate B, Nazir S, Alemayehu A. Cystic echinococcosis amongst small ruminants and humans in central Ethiopia. Onderstepoort J Vet Res 2015; 82 (1): E1-7.

18.- Conchedda M, Antonelli A, Caddori A, Gabriele F. A retrospective analysis of human cystic echinococcosis in Sardinia (Italy), an endemic Mediterranean region, from 2001 to 2005. Parasitol Int 2010; 59 (3): 454-9.

19.- Brundu D, Piseddu T, Stegel G, Masu G, Ledda S, Masala G. Retrospective study of human cystic echinococcosis in Italy based on the analysis of hospital discharge records between 2001 and 2012. Acta Trop 2014; 140: 91-6.

20.- Moldovan R, Neghina A M, Calma C L, Marincu I, Neghina R. Human cystic echinococcosis in two south-western and central-western Romanian counties: a 7-year epidemiological and clinical overview. Acta Trop 2012; 121 (1): 26-9.

21.- Ernest E, Nonga H E, Kynsieri N, Cleaveland S. A retrospective survey of human hydatidosis based on hospital records during the period 1990-2003 in Ngorongoro, Tanzania. Zoonoses 
Public Health 2010; 57 (7-8): e124-9.

22.- Schenone H, Contreras M, Salinas P, Sandoval L, Saavedra T, Rojas A. Hidatidosis humana en Chile. Seroprevalencia y estimación del número de personas infectadas. Bol Chil Parasitol 1999; 54 (3-4): 70-3.

23.- Karim M F, Brunetti E, Rahman S, Budke C M, Ahsan A S, Al-Mahtab M, et al. Abdominal cystic echinococcosis in Bangladesh: a hospital-based study. J Infect Dev Ctries 2015; 9 (1): 70-5.

24.- Stiglich M, Vega-Briceño L, Gutiérrez $M$, Trefogli P, Chiarella P. Hidatidosis pulmonar pediátrica: Reporte de 12 años de experiencia.
Rev Chil Pediatr 2004; 75 (4): 333-8.

25.- Fakhar M, Sadjjadi S M. Prevalence of hydatidosis in slaughtered herbivores in Qom Province, central part of Iran Vet Res Commun. 2007; 31 (8): 993-7.

26.- Elmahdi I E, Ali Q M, Magzoub M M A, Ibrahim A M, Saad M B, Romig T. Cystic echinococcosis of livestock and humans in central Sudan. Ann Trop Med Parasitol 2004; 98 (5): 473-9.

27.- Njoroge E M, Mbithi P M F, Gathuma J M, Wachira T M, Gathura P B, Magambo J K, et al. A study of cystic echinococcosis in slaughter animals in three selected areas of northern
Turkana, Kenya. Vet Parasitol 2002; 104 (1): 85-91.

28.- Abdel-Hafez S K, Al Yaman F M, Said I M. Further-studies on prevalence of hydatidosis in slaughtered animals from North Jordan. Z Parasitenkd 1986; 72 (1): 89-96.

29.- Sotiraki S, Himonas C, Korkoliakou P. Hydatidosis-echinococcosis in Greece. Acta Trop 2003; 85 (2): 197-201.

30.- Ernest E, Nonga H E, Kassuku A A, Kazwala R R. Hydatidosis of slaughtered animals in Ngorongoro district of Arusha region, Tanzania. Trop Anim Health Pro 2009; 41 (7): 1179-85. 